\title{
From Lunar Regolith To Fabricated Parts: Technology Developments and the Utilization of Moon Dirt
}

\author{
C. A. McLemore ${ }^{1}$, J. C. Fikes 2 , K. S. McCarley ${ }^{3}$, J. E. Good ${ }^{4}$, S. D. Gilley ${ }^{5}$, and J. P. \\ ${ }^{1}$ National Aeronautics and Space Administration (NASA), Marshall Space Flight \\ Center, Alabama, 35812; PH (256) 544 2314; FAX (256) 544 5853; email: \\ carole.a.mclemore@nasa.gov \\ ${ }^{2}$ National Aeronautics and Space Administration (NASA), Marshall Space Flight \\ Center, Alabama, 35812; PH (256) 544 5570; FAX (256) 544 5853; email: \\ john.c.fikes@nasa.gov \\ ${ }^{3}$ National Aeronautics and Space Administration (NASA), Marshall Space Flight \\ Center, Alabama, 35812; PH (256) 544 2298; email: kevin.s.mccarley@nasa.gov \\ ${ }^{4}$ Teledyne Brown Engineering, Huntsville, Alabama, 35805; PH (256) 726 1199; \\ email: jim.good@tbe.com \\ ${ }^{5}$ Tec-Masters, Inc., Huntsville, Alabama, 35806; PH (256) 726 2251; email: \\ sgilley@tecmasters.com \\ ${ }^{6}$ Teledyne Brown Engineering, Huntsville, Alabama, 35805; PH (256) 544 7016; \\ FAX (256) 5445853 email: jim.kennedy@tbe.com
}

\footnotetext{
ABSTRACT

The U.S. Space Exploration Policy has as a cornerstone the establishment of an outpost on the moon. This lunar outpost will eventually provide the necessary planning, technology development, testbed, and training for manned missions in the future beyond the Moon. As part of the overall activity, the National Aeronautics and Space Administration (NASA) is investigating how the in situ resources can be utilized to improve mission success by reducing up-mass, improving safety, reducing risk, and bringing down costs for the overall mission. Marshall Space Flight Center (MSFC), along with other NASA centers, is supporting this endeavor by exploring how lunar regolith can be mined for uses such as construction, life support, propulsion, power, and fabrication. An infrastructure capable of fabrication and nondestructive evaluation will be needed to support habitat structure development and maintenance, tools and mechanical parts fabrication, as well as repair and replacement of space-mission hardware such as life-support items, vehicle components, and crew systems. This infrastructure will utilize the technologies being developed under the In Situ Fabrication and Repair (ISFR) element, which is working in conjunction with the technologies being developed under the In Situ Resources Utilization (ISRU) element, to live off the land. The ISFR Element supports the Space Exploration Initiative by reducing downtime due to failed components;
} 
decreasing risk to crew by recovering quickly from degraded operation of equipment; improving system functionality with advanced geometry capabilities; and enhancing mission safety by reducing assembly part counts of original designs where possible. This paper addresses the need and plan for understanding the properties of the lunar regolith to determine the applicability of using this material in a fabrication process. This effort includes the development of high fidelity simulants that will be used in fabrication processes on the ground to drive down risk and increase the Technology Readiness Level (TRL) prior to implementing this capability on the moon. Also discussed in this paper is the on-going research using Electron Beam Melting (EBM) technology as a possible solution to manufacturing parts and spares on the Moon's surface.

\section{INTRODUCTION: THE NEW CALL TO EXPLORATION}

The U.S. Space Exploration Policy explicitly mades mention of utilizing the resources of the Moon and the planets beyond. This concept of making use of the resources found in situ was further expanded in NASA's Exploration Systems Architecture Study (2005) and the refinements of the lunar architecture that came after. With a plan to establish a long-term presence on the moon "to stay", unlike past one-at-a-time mission approaches, establishing capabilities to make use of resources present will offer distinct advantages in up-mass and overall cost.

As a step in accomplishing this Policy, NASA established a coordinated effort for ISRU, managed at Johnson Space Center (JSC) and including the participation of multiple NASA centers. The Marshall Space Flight Center (MSFC) is an ISRU project team member and leverages experience from previous research and experience in this area. The first aim of ISRU is to utilize available resources to produce oxygen on the moon for use as propellants and, possibly, life support. Much work is on-going to exploit the lunar regolith to extract this precious gas, and also other volatiles. As part of the necessary infrastructure to support this extraction of volatile resources, considerable quantities of the regolith will need to be mined and processed. Metals and other materials can be captured as by-products from the spent regolith. These materials can be used as feedstock for other applications.

It was recognized by NASA early in the planning of the new lunar architecture that, if humans were to inhabit spaces far removed from earth for extended times, new approaches would have to be developed to conquer the old problems of keeping hardware operational through an adequate supply of spare parts. Rather than transporting from Earth a full spectrum of all the replacement parts that might reasonably be needed by a lunar base, one approach studied was to manufacture the parts locally (i.e., in situ). Thus, only the mass of adequate raw materials for parts fabrication, and the machinery to do so, would need to be transported, rather than a full catalog of replacement parts. Better yet, if the raw materials could be provided locally, then that needed up-mass could be eliminated as well. Thus, the desires for an in situ fabrication capability converged with the need for an in situ resource utilization capability.

In addition to decreasing the required up-mass and thus the life-cycle costs of the mission architecture, it was seen that an in situ manufacturing capability would reduce risk for the crew and the mission. In the event of failure or eminent failure of critical parts for which no spares are available on site, resupply from Earth would 
take at least three days, even assuming that a very expensive "launch on need" capability was maintained on Earth. With a fabrication capability at the lunar base, parts could be built in a very short time, potentially saving a mission, or even lives. The ISFR team at MSFC studied the options and the requirements for a fabrication capability and selected a small number of additive fabrication processes showing great promise to pursue for development of the technology. One of these methods, Electron Beam Melting (EBM), was selected as the best candidate for a wide range of potential lunar applications and parts, utilizing a range of metals. MSFC purchased an EBM machine in June 2006 and has been acquiring experience on the machine by making titanium parts. MSFC has captured knowledge of proper machine theme settings, correct part orientations, and other requirements necessary to successfully manufacture parts that can meet ASTM standards. Post-build part finishing (machining) has also been investigated, and tests have been performed on both unfinished and finished part.

As part of the investigation into the requirements for such a fabrication capability, it was recognized that other complementary and supporting capabilities and technologies would have to be developed. Among these, it was recognized that as a natural follow-on it would be necessary to adequately verify the parts that are built in situ. A method of inspecting and analyzing the as-built part would be needed, and preferably a method that would not require any type of destructive testing or. degradation of the part. Nondestructive evaluation (NDE) techniques would therefore be needed, and some required varying levels of technology development as well. As an added benefit, these same NDE techniques would be capable of aiding in preventative maintenance and of detecting flaws and other problems in structures that were not built with the in situ manufacturing capabilities on the Moon, but rather had been brought from Earth.

The MSFC ISFR team has prepared a plan to deal with these details from the extraction of resources from regolith to the production of operations-ready parts at a lunar base. Considerable development of technologies must be accomplished in several areas to make this a reality, but there are benefits along the way that pay off in the near term, as well as the long-tem goals of mitigating risk, reducing up-mass, improving reliability, and reducing life-cycle costs. An overview of this plan is shown in Figure 1.

\section{UNDERSTANDING REGOLITH}

Very limited amounts of the lunar soil were returned from the six Apollo missions that landed on the lunar surface in the 1960s and 1970s. A total of only 382 kilograms of rocks, core samples, sand and dust were returned. Three automated Soviet spacecraft returned samples totaling about 300 grams from three other sites. This source material has been extensively studied, but mysteries regarding the properties remain, though better data has been gathered from the samples as test equipment has become more technologically advanced. However, these samples represent only nine locations on the lunar surface. Assuming that analysis of these samples would reveal everything about lunar dirt would be the equivalent of taking soil samples from six locations on Earth and assuming that everything about the planet's soil could be deduced from these. 


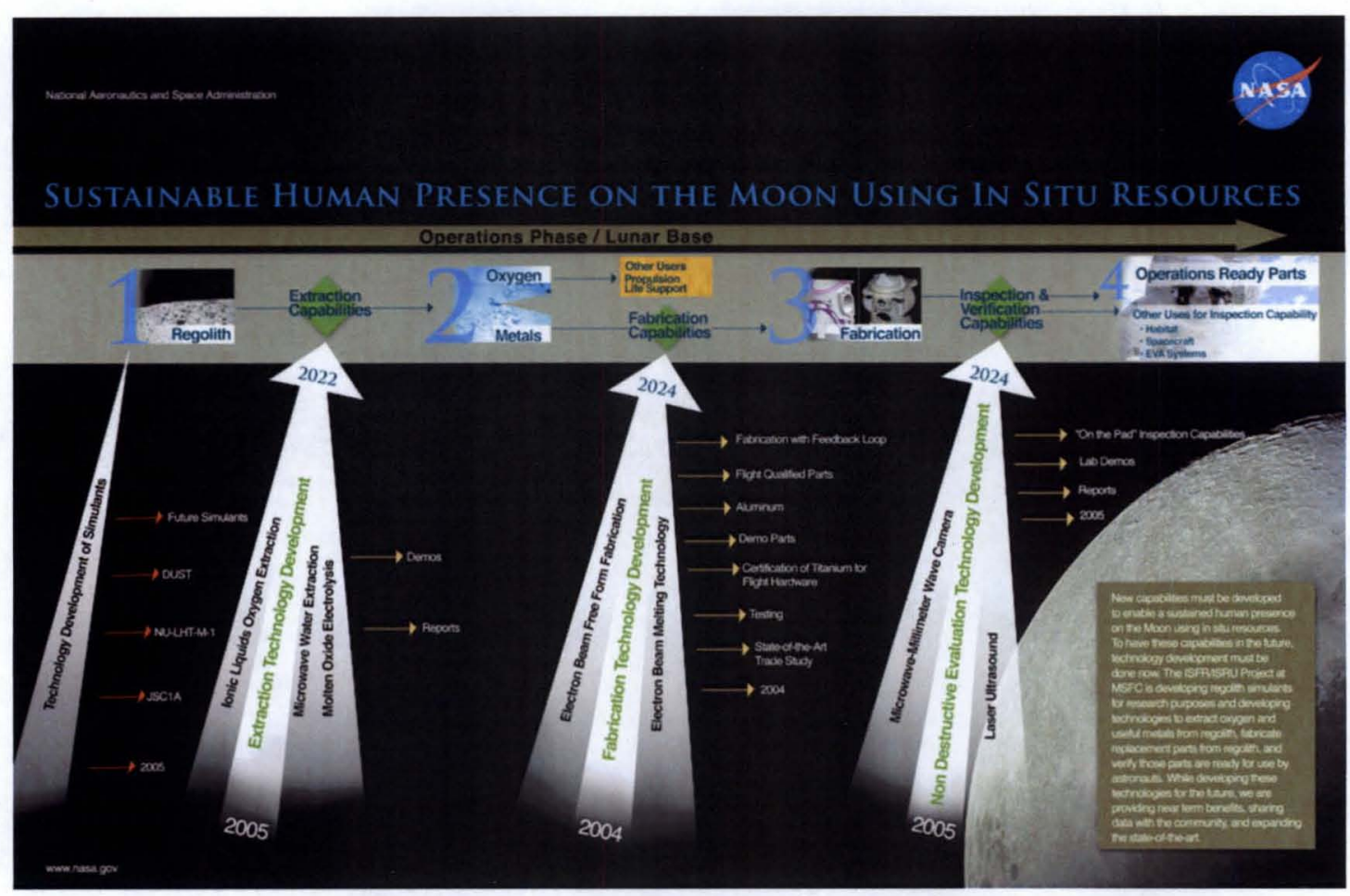

Figure 1. Use of lunar in situ resources

In the years since the Apollo missions, various simulants of lunar soil were produced to allow experimentation on material as close as possible to "the real thing." Two such simulants produced in the 1980s and 1990s were MLS-1 (the University of Minnesota) and JSC-1 (NASA Johnson Space Center). (Both of these were designed to simulate the lunar mare regions.) Though the different simulants were designed and produced for different purposes, JSC-1 became something of a de facto standard among simulants.

With the advent of the new U.S. Space Exploration Policy and an increased interest in exploitation of lunar resources, the remaining supply of JSC-1 was quickly depleted. Working under the NASA Human Systems Research and Technology Division, the MSFC ISRU project team was given the lead for production of a new range of lunar simulants, beginning with production of a quick "replacement" for JSC-1, having properties close to that simulant. This new simulant, named JSC-1A, was produced starting in 2006.

Other simulants followed, including those developed by private companies as well as international partners. The most recent simulants being developed under the leadership of MSFC include the NASA/U.S. Geological Survey Lunar Highland Type (NU-LHT) simulants and a pilot dust simulant. While these simulants are currently being developed for the ISRU and Dust Management Projects under the Exploration Technology Development Program (ETDP) in rather small quantities, plans are to ramp up production to meet multiple users' needs including the Constellation Project as well as other ETDP projects. "Designer" simulants will be developed as required in order to meet users' specific properties needs. MSFC has been contacted by many organizations within NASA, industry and educational partners, for purposes of 
acquiring a NASA simulant that can be used in testing of various processes and for other reasons including education.

For specific purposes, different properties of the lunar soil need to be mimicked by the simulants. For example, for experiments involving candidate methods of oxygen extraction, chemical composition of the simulant samples is vitally important, as well some morphological characteristics of the soil grains. On the other hand, for tests involving how the wheels of proposed vehicle types would behave in the lunar soil, chemical composition is less consequential, but gross physical characteristics are vitally important.

By their nature, no simulant will ever be a "perfect" replication of lunar regolith. Even if all the properties of the lunar soil were characterized, and even if the lunar region to be simulated was limited to a single site, no simulant could be produced that would mimic all the properties of the lunar soil. Base material that is identical to the lunar material simply does not exist on Earth. As an example, it is extremely difficult to produce on Earth grains with the same jagged characteristics of lunar soil, which has not been exposed to the weathering effects of wind and water for hundreds of millions of years, at least. Even if it did, most uses of the soil would necessarily expose it to the atmosphere at some point - this would of necessity make changes in the soil, including the absorption of water.

The MSFC ISRU group has been working with the user community, including through a series of Workshops, to develop simulants that meet the needs of those performing development and eventually verification and certification testing. The most recent of these workshops was held in Huntsville, Alabama in October 2007. These have helped in defining the properties that must be matched by the simulants, the different needs of accuracy for these parameters, the variations of simulants that need to be produced to meet the users' needs, and the requirements for quantities of the various simulants. By keeping the source for lunar regolith simulants centralized, rather than encouraging each user to produce their own to meet their own particular needs, NASA can defray the costs of development. In addition, a standard simulant can "level the playing field" among competing technologies (e.g., different methods of oxygen extraction) by allowing an "apple to apples" comparison of test results, since they are working with the same starting material. If desired, of course, a user can utilize another simulant(s) in their research. In order to make fair comparison across research results, the Figures of Merit developed by MSFC will be invoked which equates an evaluation of the simulant against other simulants or against actual Apollo samples.

As more is known about the lunar regolith through continued examination of samples returned by Apollo and through data returned by future lunar missions, such as the Lunar Reconnaissance Orbiter and the Lunar Crater Observation and Sensing Satellite (LCROSS), these simulants can be continually refined. Furthermore, as the user community gets "smarter" about their processes and knows better what their needs are, the properties of the lunar simulants will be further adjusted and simulants will be "tailored" for specific needs.

\section{PRODUCTION OF OXYGEN}

Before utilizing the lunar regolith for any purposes, sizeable quantities of it will need to be excavated. In many cases, equipment can be used for dual purposes, which 
means that this equipment will not add an excessive amount of mass to be able to execute ISRU. Some concepts still being refined involve the use of mobile processing equipment, capable of moving to the site where the mining occurs. In most concepts, though, this regolith will also need to be transported to the location where it will be processed. These mining and transportation functions are being addressed by elements related to ISRU.

Once the material has been mined and transported to the processing plant for extraction of volatiles, a certain degree of pre-processing must occur. This process, known as beneficiation, may involve milling, material separation or other processes, depending on the method used for oxygen extraction and extraction of other materials. This will change the regolith into a physical form that is easier to process, and in most cases increase the concentration of desired elemental fractions. The material removed may simply be discarded as "waste", or it may be used for other purposes, such as construction of berms, bricks, or other physical structure, or as radiation shielding materials.

Several different oxygen extraction processes are being studied by different entities for potential use on the lunar surface. The field of candidate methods has already been narrowed and, as the technologies mature and further research is done, the field will be narrowed even further. Eventually, one or more technologies will be developed for field tests on the lunar surface (pilot plants), and one method will be selected to be used in the production of oxygen for future missions. Among the technologies for extraction of oxygen from regolith currently being developed at the Marshall Space Flight Center are two methods of electrolysis, one utilizing a high temperature molten oxide and the other utilizing acidic ionic liquids. In the case of molten oxide electrolysis, the beneficiated regolith is heated to a high temperature and oxygen is produced at the anode, with metals produced at the cathode. Experiments have been conducted at temperatures up to $1650^{\circ} \mathrm{C}$ and have shown the feasibility of this method. Because of the high temperatures involved and the resultant heat loss expected, a great deal of energy is required for this method, and recycling of the heat is a priority,

In the other method, the regolith is dissolved in an acidic ionic liquid (a class of liquids having essentially zero vapor pressure). Water is produced from the oxygen bound in the metal oxides and the hydrogen from the acid. The water can be distilled and condensed into another ionic liquid electrolyte or electrolyzed within the same ionic liquid. In either case, oxygen is collected from the electrolysis of water, and the hydrogen is utilized to regenerate the ionic liquid acid. This method also shows great promise, but several technical issues are still being addressed.

Another method of oxygen production being developed at MSFC makes use of the water ice that is conjectured to be trapped below the lunar surface in the permanently shadowed craters at the poles. (Data from the Clementine probe in 1994 and the Lunar Prospector in 1999 gave indications of such permafrost, but its presence remains unconfirmed. The LCROSS mission will help to confirm or refute these findings.) Early manned missions are planned for the lunar South Pole in part to exploit this resource. Experiments have been conducted with simulant to show that microwaves can be used to heat the lunar regolith adequately to produce enough vapor pressure to cause the gaseous water to rise to the surface. Microwaves will 
penetrate a meter or more into the regolith, allowing this method to be used without digging or disturbing the lunar surface. After the water vapor is collected, it can be electrolyzed to produce gaseous oxygen and hydrogen. Utilization of this method means that adequate hydrogen would be produced on the Moon to fuel the ascent vehicles, thus eliminating the need for this to be staged from Earth. This method, though, relies in the uncertain presence of permafrost and on working in the extreme cold of the permanently shadowed lunar craters. This also does not produce any metallic byproducts for use as raw materials in a fabrication process.

For each of these methods, technology development proceeded utilizing the available regolith simulants. Experiments have been conducted to show the feasibility of each of these methods, but further technology development work is needed. In each case, the methods used will need to be scaled up to show their feasibility on a mass production scale. As further testing reveals which methods or methods will be most useful and efficient under lunar conditions, project devices will need to be designed and built so that they can be tested in simulated conditions (and possibly in pilot programs on the lunar surface) before a selection can be made for a method to produce oxygen for actual use in situ.

\section{PARTS PRODUCTION: ART TO PART}

As previously noted, some methods of oxygen extraction under study produce considerable quantities of "spent" lunar regolith that have been enriched in metals such as aluminum and titanium. These are valuable resources for the production of replacement and new parts for the growing lunar base. Initially, in the early days of a lunar base, it will not be feasible to utilize the lunar regolith as a base material. The EBM process can produce parts in situ utilizing provisioned feedstock, which can yield a sizeable reduction in the up-mass required for the typical payload of spare parts. This would result in a significant cost savings as well. As noted previously, it also has a benefit in risk reduction. It is inevitable that components will fail and require repair or replacement, and new designs will require tooling and finished parts. A fabrication capability on the lunar surface will address these needs and is critical. The current focus for the production of metallic parts in situ utilizes the Electron Beam Melting (EBM) process. This technology uses an electron beam in a vacuum to melt metal powders layer-by-layer. The build results in a near-net shape part that is fully dense. In other words, the part does not have the voids and pits found in similar powder-based additive manufacturing process. Mechanical testing of titanium samples has shown good strength properties as well as fracture and fatigue. Results show the properties exceed those of similar cast materials and are comparable to wrought values. This is very promising data and validates the decision to concentrate on the EBM process.

The process begins by providing a Computer-Aided Design (CAD) file to the machine. This file is processed by slicing the CAD model into horizontal layers. Each layer defines a two-dimensional path that the EBM gun will raster onto the powder bed. After the layer has been sintered, the powder bed is lowered by the thickness of one layer and then a rake system spreads a new layer of powder over the previous one. Each layer is melted and becomes an integral part of the previous layer until the part is completed. Having CAD models for existing parts reduces the time it takes to fabricate a part; however, new designs can be fabricated as quick as $\mathrm{CAD}$ models can 
be produced. Designers have more freedom in that complex geometries can be utilized, geometries that cannot be produced by traditional manufacturing techniques. Internal cavities can be fabricated. Assemblies can be fabricated with fewer parts because they can be combined in the design phase, knowing additive fabrication techniques are capable of producing them. In situ fabrication on the lunar surface can provide a quick turn-around for parts repair, replacement, or fabrication of new designs.

Through fabrication technology trade studies performed at the MSFC, it was determined that no one fabrication process can "do it all". For several reasons, it is anticipated that the optimum approach for fabrication on the moon will involve a hybrid system where a combination of additive and subtractive technologies would be used to arrive at the most effective fabrication capability. More research will need to be performed in order to determine the best suite of equipment and technologies. The EBM technology is highly versatile in the number of metallic materials that can be processed: Different industries are interested in differing materials for their specific applications. For aerospace applications titanium is a popular choice for material but may also include interest in aluminum, stainless steels, and also inconels. The EBM process has shown it is capable of processing all of these materials. Currently, MSFC has concentrated their development effort on titanium, only. Aluminum is another material of interest and is undergoing an early development effort in order to make it available for EBM processing.

The MSFC ISFR team is developing the EBM technology in order to support existing programs at MSFC as well as other NASA centers and also industries across the country. Our longer-term goal is to take advantage of the EBM process to develop a system that can be utilized on the moon for in situ fabrication. Along the same lines, the military has shown an interest in fabricating parts within a war zone in order to rapidly replace failed parts. This in situ fabrication has similar requirements to remote fabrication on the moon. For this reason, the military has acknowledged a common need.

Another commonality between space applications and military applications is the need for certification of the process. MSFC has teamed with the Boeing Company to initiate a certification effort that will provide a process specification to fabricate space-certified parts. The military will need this type of certification effort in order to produce valid parts for use on existing military hardware. The required testing has been completed and is being documented and used to validate the process and to determine acceptable parameters for the EBM machine. Initial analysis of the data has shown good results and final test results are being analyzed. Results will be implemented into the existing specification (AMS 4999).

It is planned that initial parts produced on the lunar surface will be built from a powder stock that is provisioned from Earth. As noted, this can provide a significant mass savings, through elimination of many spare parts that would otherwise have to be brought from Earth, and through the fact that parts built additively require significantly less material than those built subtractively. (The EBM process is efficient where the unused powder can be recycled and used in subsequent builds.) However, to realize the maximum mass advantage, locally produced materials (i.e. lunar regolith) will eventually be utilized. 
As previously noted, extracting oxygen from lunar regolith can result in a useful byproduct that is relatively enriched in metals. This byproduct can be processed and developed into a useful feedstock for in situ fabrication. Processing would include producing grains of more uniform size and separating materials for optimum and consistent mixes. The most advantageous approach for timer and energy would be to use lunar regolith as mined or only mechanically processed. Based upon preliminary analysis, it is believed that the regolith would require little or no pre-processing before use as an EBM feedstock; however, a binding material (possibly $10 \%$ by mass) would need to be added to allow the regolith to properly melt and allow a part to be built with sufficient strength.

As a simple test run, some NASA/U.S. Geological Survey Lunar Highland TypeMedium NU-LHT-1M lunar regolith simulant was used recently as feedstock in an EBM machine. The common assumption was that the simulant in this trial run would not melt, and the beam would blow the material away from the build plate. This test was seen as valuable to provide baseline data. Surprisingly, a light sintering did occur with the pure regolith simulant. While this was a promising start, more work must be done before definitive determinations are made. Subsequent development efforts will concentrate on determining the minimum mix of binder material required to successfully melt raw regolith into an "adequate" solid material. Additionally, metals known to be found in the lunar regolith will be investigated to determine how viable they are for EBM processing, thus determining their value for in situ fabrication on the lunar surface.

\section{ACCEPTANCE OF PARTS}

To produce parts for spaceflight, whether on Earth or the lunar surface, the EBM process needs to be certified, as noted. In addition, parts as built will need to be verified for a host of properties. While this is easily done for parts built on Earth (through building additional parts for various strength tests and performing other tests on conventionally built parts), the task is more complicated for parts produced on the lunar surface, as equipment will not be present to perform the myriad of tests that can be done in a terrestrial laboratory.

Thus, methods of nondestructive evaluation (NDE) that can be performed with portable or even handheld equipment and able to perform in lunar environments must be developed. The MSFC ISFR team performed trade studies to select technologies that would be of benefit for these verifications and would also serve other purposes, such as detection of flaws in other structures.

One of the methods currently in development by the MSFC ISFR team utilizes laser ultrasonics. This technology shows particular promise for analyzing metallic parts built on the moon due to several factors, among them being no need for contact, the ability to work from one side or both sides of a target, and the ability to work in an atmosphere or a vacuum. This technique utilizes one laser to create a mechanical pulse in a target material, and a second laser to detect deflection of the surface caused by the resulting elastic waves that have propagated through the target. Reflected laser light is captured, and variations in the signal produced by matching this light against a reference beam in an interferometer are used to detect any surface or subsurface irregularities in the target material. 
A laboratory laser ultrasonic device was acquired by MSFC in 2007, and an initial study was undertaken to determine what the requirements would be to eventually package it into a handheld system that could be used on parts produced through in situ fabrication, and also on structures such as spaceships and habitation modules. This initial study resulted in confirmation of the potential of a laser ultrasonic system on the moon, and recommendations for steps that need to betaken to further develop the technology.

\section{CONCLUSIONS}

As part of the U.S. Space Exploration Policy, NASA plans to return to the Moon to establish a permanent human presence, eventually leading to missions beyond the Moon. A lunar outpost will be built to sustain and accommodate the crew. Although much hardware, supplies, consumables, and other items will need to be launched from Earth, it is imperative that humans learn to "live off the land" in the long run, especially if missions beyond the Moon are to be achieved. It would be cost prohibitive to launch every single item needed, or possibly needed. Since the Moon is comprised of valuable resources, it only makes sense to "mine the Moon". The lunar regolith contains abundant resources that could be used to help sustain life on the Moon. In addition to oxygen, other materials such as metals and volatiles are present that could be used for life support, propulsion, and fabrication of habitats, solar arrays, hardware, and tools. Missions to the Moon and beyond will need to take advantage of the in situ resources to be affordable or even feasible. Thus, technologies need to be developed to extract oxygen, water (if present), and metals from the regolith. Fabrication technologies will also need to be developed and advanced in order to utilize the resources as feedstock to build spares for parts that break on the Moon or to build tools, structures, or other new items not envisioned before launching from Earth. After the items are fabricated, it will be necessary in most cases to inspect them to ensure part integrity and safety. This calls for nondestructive evaluation technologies to also be available for use on the Moon. The NASA MSFC is contributing to the advancement of these technologies through studying and understanding the lunar regolith and its properties in order to develop lunar simulants for use in human toxicology studies as well as hardware development and verification purposes. Recent lunar simulants that have been developed and/or are in process of being developed by MSFC with various partners include the JSC-1A (mare type simulant) and the NU-LHT (NASA/U. S. Geological Survey Lunar Highland type simulant) with more types such as a dust simulant currently in development. In addition, MSFC has been advancing the certification process for materials used with Electron Beam Melting. This fabrication technology has not only long-term uses in space, but also near-term uses on Earth in the medical, military, and commercial fields. Laser ultrasound technology research is also being performed by MSFC that will complement the fabrication technology by providing a means to perform non-invasive inspections of hardware that have been fabricated. Again, this technology could be utilized on Earth as well in space. To round out MSFC's suite of ISFR \& ISRU technology research, MSFC has investigated the possibility of extracting oxygen on the Moon using ionic liquids and molten oxide electrolysis, and using microwaves to extract water, assuming it is present. All these technologies 
show great promise but require additional development to be ready to help achieve the goals of the Space Exploration Initiative. 